\title{
SEXISMO Y JUSTIFICACIÓN DE LA VIOLENCIA EN ADOLESCENTES ECUATORIANOS DE CONTEXTOS EN RIESGO PSICOSOCIAL
}

\author{
Sexism and justification of violence among Ecuadorian \\ adolescents in psychosocial risk contexts
}

\author{
Rocío Soto Bustamante* \\ Pontificia Universidad Católica del Perú
}

Perú

\begin{abstract}
Resumen
La investigación tiene como propósito analizar las relaciones entre el sexismo ambivalente y las actitudes de justificación de la violencia (entre iguales y doméstica) en 73 adolescentes (43 mujeres y 30 varones), de 11 a 18 años $(M=15.74, D E=1.14)$, de una comunidad en riesgo psicosocial de Loja, Ecuador. Se utilizó el Cuestionario de Actitudes hacia la Diversidad y la Violencia (Díaz-Aguado, Martínez Arias, \& Martín Seoane, 2004) y el Inventario de Sexismo Ambivalente para Adolescentes (De Lemus et al., 2008). Se obtuvieron relaciones positivas entre las variables, donde el sexismo hostil demostró ser un predictor fuerte de las actitudes que justifican la violencia doméstica $(R 2=.40, p<.001)$ y la violencia entre iguales $(R 2=.39$, $p<.001)$. Por tanto, resulta relevante para las comunidades en riesgo, desarrollar programas preventivos de la violencia que promuevan una cultura de paz y la deconstrucción del sexismo, a través del fortalecimiento de sus derechos y potenciación de recursos.
\end{abstract}

Palabras clave: sexismo, violencia, género, adolescentes en riesgo psicosocial.

\begin{abstract}
The research aims to analyze the relationships between ambivalent sexism and attitudes justifying violence among peers and domestic violence (in 73 adolescents (43 females and 30 males) aged from 11 to 18 years $(M=15.74, S D=1.14)$, from a community in psychosocial risk in Loja, Ecuador. The Attitudes towards Diversity and Violence Questionnaire (Díaz-Aguado, Martínez Arias, \& Martín Seoane, 2004) was used, as well as the Inventory of Ambivalent Sexism for Adolescents (De Lemus et al., 2008). Positive relationships were obtained between the variables, where hostile sexism proved to be a strong predictor of attitudes that justify domestic violence $(R 2=.40, p<.001)$ and violence between peers $(R 2=.39, p<.001)$. Therefore, on the one hand, it is relevant for communities at risk to develop violence prevention programs that promote a culture of peace and, on the other hand, the deconstruction of sexism by strengthening of their rights and empowerment of resources.
\end{abstract}

Keywords: Sexism, Violence, Gender, Adolescents in psychosocial risk.

\footnotetext{
Cualquier uso que se haga de este artículo debe incluir: Autor / Título original de la publicación / ISSN.
} 


\section{INTRODUCCIÓN}

La violencia más que una problemática se podría considerar un indicador debido a su causalidad multifactorial y estructural (Marchioni, 2001), que afecta en mayor proporción a la población de niñas, niños, adolescentes y mujeres del mundo.

Específicamente en Ecuador, en el 2012, se reportaron 17370 casos de niñas, niños y adolescentes que sufrieron algún tipo de violencia, entre negligencia (42\%), psicológica (25\%), física (18\%) y abuso sexual (7\%) (UNICEF, 2014). En el caso de mujeres jóvenes y adultas, los reportes indican que el $48.7 \%$ habría sufrido algún tipo de violencia por parte de su pareja o ex pareja, destacando la violencia psicológica (43.4\%). Dicha tendencia alarmante de la problemática de violencia contra la mujeres en la relación de pareja se replica en la provincia de Loja, donde se encuentran los siguientes indicadores: violencia psicológica (41.4\%), física (31.1\%), sexual (12.2\%) y patrimonial (7.8\%) (Instituto Nacional de Estadísticas y Censos de Ecuador, 2018). Concretamente en el barrio "Víctor Emilio Valdivieso", se evidencian indicadores de riesgo psicosocial tales como violencia intrafamiliar, violencia contra las mujeres, consumo de drogas, embarazo adolescente, robos y conflictos comunitarios, que impactan en el desarrollo de sus pobladores, sobre todo en la población infantil y adolescente (Aguirre et al., 2019). Por lo que resulta relevante generar espacios de vida saludable con adolescentes de la comunidad, a fin de reducir los factores de riesgo, sobre todo en materia de violencia de pareja y violencia intrafamiliar.

$\mathrm{Al}$ respecto, diversos estudios destacan entre los factores que pueden predecir la violencia de pareja, al sexismo ambivalente y las actitudes que justifican la violencia (Arnoso et al., 2017; Ibabe et al., 2016; León-Ramírez y Ferrando, 2014).

\section{SEXISMO AMBIVALENTE}

Desde la conceptualización de Glick y Fiske (1996), el sexismo ambivalente puede ser entendido como el resultado de la combinación de dos elementos con cargas afectivas antagónicas, que son consecuencia de las complejas relaciones de aproximación/evitación que caracteriza a los sexos. La ambivalencia sexista emerge de la influencia simultánea de dos tipos de creencias sexistas, que marcan una tendencia a responder de forma muy favorable o totalmente desfavorable. En ese sentido, el Sexismo Hostil, se aplica como un castigo a las mujeres no tradicionales, puesto que estas no asumen los roles de género tradicionales e intentan modificar las relaciones de poder entre hombres y mujeres. Por su parte, el Sexismo Benevolente resulta una recompensa para las mujeres que cumplen con los roles tradicionales, ya que éstas aceptan la supremacía masculina.

En relación a las diferencias por género, cierta evidencia posiciona a los hombres con un nivel mayor de sexismo hostil (Ferragut et al., 2017), mientras que las mujeres reportarían un mayor sexismo benevolente (Austin \& Jackson, 2019; Jitaru, \& Holman, 2017; Rottenbacher, 2010). El sexismo hostil es la forma de discriminación más explícita y obvia, en contraste al sexismo benevolente que resulta más implícito y sutil por lo que es más proclive a ser aceptado por las mujeres, especialmente en culturas con mayor incidencia de abuso físico y psicológico (Lee et al., 2010).

Bajo este contexto, la perspectiva psicosocial del género otorga al sexismo la responsabilidad de ser una de las principales creencias que mantiene las desigualdades entre hombres y mujeres, evidenciándose así su relación con ciertas formas de violencia hacia las mujeres (Archer, 2006). En esa línea, el sexismo y la aceptación de la violencia en las relaciones sociales estarían positivamente asociados con la aceptabilidad de la violencia íntima de pareja, siendo que los niveles más altos de dicha aceptabilidad se hallaron en las personas con mayores puntuaciones de sexismo, las cuales también presentaron actitudes positivas hacia el uso de la violencia en las relaciones sociales (Herrero et al., 2017).

Asimismo, las creencias sexistas se relacionarían positivamente con la victimización en las relaciones de pareja, destacando el sexismo hostil como su principal predictor (Ibabe et al., 2016). Por su parte, el sexismo benevolente estaría relacionado con una mayor atribución de culpa hacia la víctima en el caso de una violación (Durán et al., 2010).

Finalmente, Ramiro-Sánchez et al. (2018) hallaron que los adolescentes que presentan más actitudes 
sexistas reportan actitudes más positivas hacia la violencia de pareja, más atracción por parejas sexistas, mayor apoyo hacia el mito idealizado del amor y de vinculación amor maltrato, mayor dependencia emocional en la pareja y peor calidad en las relaciones de pareja.

\section{ACTITUDES DE JUSTIFICACIÓN DE LA VIOLENCIA}

Para la psicología social el término actitud podría definirse como la evaluación subjetiva que realizamos de una persona, producto o grupo social (Albarracín et al., 2005) la cual considera tres componentes: cognitivo, afectivo y comportamental (Jhangiani, \& Tarry, 2014).

El componente cognitivo vendría a ser el estereotipo, conjunto de creencias positivas o negativas que tenemos sobre las características de un grupo social. Para el caso de las actitudes negativas, el estereotipo estaría definido por las creencias negativas (Jhangiani et al., 2014). El prejuicio sería considerado el componente afectivo y se lo define como una actitud irrespetuosa e injustificable hacia un grupo social. Este puede tomar la forma de enojo, miedo, e incomodidad, siendo estados afectivos que pueden conducir a comportamientos (Jackson, 2011). Bajo este contexto, los estereotipos y prejuicios serían problemáticos ya que pueden crear discriminación o tendencias de acción negativas hacia determinadas personas o grupos sociales (Jhangiani et al., 2014).

En esa línea, las creencias y actitudes que justifican el uso de la violencia contra las mujeres pueden constituir uno de los factores de riesgo para que se produzca el maltrato en la pareja. Al respecto, existiría dos condiciones de riesgo de especial relevancia: la tendencia a justificar y reproducir los modelos sexistas y violentos de la infancia y la adolescencia; y el desequilibrio de poder existente entre los varones y las mujeres, a partir del cual se originan y perpetúan los estereotipos vinculados al género (Heise et al., 2002).

En el presente estudio, la justificación de la violencia se define a partir de las actitudes que apoyan el uso de la violencia en ciertas situaciones: 1) justificación de la violencia entre iguales como reacción y valentía, asociada a la creencia de que el uso de la violencia puede estar justificado y que la demostración de violencia es positiva; y 2) la justificación de la violencia doméstica, que se refiere a la justificación de la dominancia patriarcal de la familia, de la discriminación sexista y de la violencia ejercida contra mujeres, niñas y niños (Díaz-Aguado et al., 2004).

La justificación de la violencia estaría asociada a ciertas variables sociodemográficas como el género, nivel de educación y edad. Según Sardinha y Nájera (2018) las mujeres en el África subsahariana y el sur este de Asia son más propensas a justificar la violencia de pareja en sus relaciones. Mientras que en adolescentes, Ferragut et al. (2017), Garaigordobil et al. (2013b) hallaron en España que son los varones los que tienden a justificar más la violencia. Similares resultados se obtuvieron en Ecuador, donde los adolescentes con una historia de consumo de alcohol en la familia, violencia entre hermanos y un alto grado de disfrute de la violencia en los medios, reportaron actitudes más fuertes de justificación de la violencia entre compañeros, violencia doméstica y sexual (Pinos, V., Pinos, G., Jerves, \& Enzlin, 2016).

Respecto al nivel de educación, entre las mujeres, las mayores tasas nacionales de alfabetización femenina pronosticaron niveles más bajos de justificación. En cuanto a la edad, se observó que la justificación de la violencia doméstica y contra minorías disminuyó con el aumento de la edad durante el curso escolar, sin embargo, en lo que respecta a la justificación de la violencia entre iguales no hubo cambios en relación a esta variable (Garaigordobil et al., 2013b).

\section{SEXISMO AMBIVALENTE Y JUSTIFICACIÓN DE LA VIOLENCIA}

Según estudios previos, el sexismo ambivalente se asociaría positivamente con la justificación de la violencia doméstica (Glick et al., 2002), comportándose como un predictor de altos niveles de esta (Yamawaki et al., 2009). Específicamente, el sexismo hostil estaría relacionado positivamente con la justificación de la violación o con actitudes menos positivas hacia las víctimas de violación (Durán et al., 2010; Sakalli-Ugurlu et al., 2007). 
En adolescentes, se observa la misma tendencia, ya que las actitudes que justifican la violencia entre iguales y la violencia doméstica se relacionan positivamente con el sexismo ambivalente, observándose que este último resulto ser un predictor positivo de dichas actitudes, donde también se destaca la mayor influencia del sexismo hostil (Fasanelli et al., 2020; Ferragut et al., 2017; Garaigordobil \& Aliri, 2013).

En las comunidades en riesgo psicosocial, el fortalecimiento de recursos y empoderamiento a través del ejercicio de los derechos son procesos que se hacen mucho más complejos y lentos si no existen lineamientos claros de intervención que sean construidos desde y para la comunidad. En tal punto, la Psicología Social Comunitaria podría aportar metodologías de intervención que dinamicen la gestión de dichos procesos, bajo un enfoque de derecho y de participación ciudadana.

En esa línea, los resultados de la investigación pretenden brindar elementos de partida para una intervención comunitaria que promueva la deconstrucción de las creencias sexistas y de actitudes que justifican la violencia, a través de la potenciación de recursos y empoderamiento de los y las adolescentes de esta comunidad. Por tanto, el estudio constituye un aporte a los Objetivos de Desarrollo Sostenible: ODS 3 de Salud y Bienestar, que busca promover el bienestar universal, y ODS 5 de Igualdad de género, que persigue eliminar todas las formas de violencia a través de la promoción de la igualdad de género y el empoderamiento.

\section{MÉTODO}

\section{Participantes}

La muestra estuvo conformada por 73 adolescentes, 43 mujeres (58.9\%) y 30 varones (41.1\%) que residen en la Ciudadela Víctor Emilio Valdivieso, Tierras Coloradas, en la provincia de Loja en Ecuador. El rango de edad fue de 11 a 18 años (M $=15.74, \mathrm{DE}=1.14)$. En cuanto al nivel de estudios, la mayoría se encuentra en el bachillerato (89\%), seguido de los niveles básico superior (6.9\%) y básico medio (4.1\%). En cuanto al tipo de familia, el $63.4 \%$ vive con ambos progenitores y hermano(a)s, el $19.7 \%$ con familia extensa y el $16.9 \%$ con familia monoparental. Por último, el 93.2\% reporta no trabajar, mientras que el $6.8 \%$ trabaja y estudia.

El acceso a los participantes se realizó como parte del proyecto de intervención que coordina la Cátedra Unesco de Cultura y Educación para la Paz de la Universidad Técnica Particular de Loja - UTPL en la comunidad.

\section{Instrumentos}

Cuestionario de Actitudes hacia la Diversidad y la Violencia $(C A D V)$ (Díaz-Aguado et al.,2004). Se utilizó el CADV para medir las actitudes de justificación de la violencia en la adolescencia. Este cuestionario consta de 56 ítems y evalúa cuatro actitudes relacionadas con la violencia y la diversidad: 1) Justificación de la violencia entre iguales como reacción y valentía; 2) Justificación de la violencia doméstica; 3) Intolerancia y justificación de la violencia hacia minorías; y 4) Acuerdo con creencias tolerantes y de rechazo de la violencia. En el presente estudio se evaluaron solo las dos primeras, haciendo un total de 33 ítems con una escala de respuesta de tipo Likert del 1 (mínimo acuerdo) al 7 (máximo acuerdo). La Justificación de la violencia entre iguales consta de 16 ítems a través de los cuales se asocia la violencia entre iguales con la demostración del propio valor. La justificación de la violencia doméstica tiene 17 ítems que justifican el dominio patriarcal de la familia, la discriminación sexista, el maltrato infantil y la violencia contra la mujer.

En cuanto a las propiedades psicométricas, respecto a la validez, se evidenciaron correlaciones entre la participación en situaciones de violencia y exclusión, y el acuerdo con creencias intolerantes y de justificación de la violencia (Díaz-Aguado et al., 2004).

En relación a la confiabilidad, la justificación de la violencia entre iguales (JVI) y de la violencia doméstica (JVD) demostraron índices altos de consistencia interna en adolescentes españoles, de .85 para cada una (Díaz-Aguado et al., 2004). En otros estudios la confiabilidad se mantiene alta, así por ejemplo Garaigordobil et al. (2013b) hallaron confiabilidades de .84 y .81 respectivamente. En 
adolescentes ecuatorianos la confiabilidad del instrumento también resulta adecuada, hallándose índices Alfa de Cronbach de .82 para JVI y de .70 para JVD (Pinos, V. et al., 2016). En el presente estudio la escala sigue evidenciando una adecuada confiabilidad, obteniéndose valores Alfa de Cronbach altos: justificación de la violencia entre iguales (.88) y justificación de la violencia domestica (.84).

\section{Inventario de Sexismo Ambivalente en} Adolescentes (ISA) (De Lemus, Castillo, Moya, Padilla, $\&$ Ryan, 2008). Es una adaptación del Ambivalent Sexism Inventory (ASI) de Glick y Fiske a la población adolescente española. El ISA-Adolescentes mide las dos dimensiones del sexismo ambivalente (SA): el sexismo hostil (SH) y el benevolente (SB), y consta de 20 afirmaciones con una escala de respuesta de tipo Likert del 1 (muy en desacuerdo) al 6 (muy de acuerdo). Un mayor puntaje en la escala indica un mayor grado de sexismo.

En relación a las propiedades psicométricas, De Lemus et al. (2008) obtuvo para una muestra de adolescentes españoles evidencias de validez. Para ello en primer lugar realizó un análisis factorial exploratorio obteniendo una adecuación de los componentes a la división del sexismo ambivalente en sexismo hostil y sexismo benévolo. Posteriormente, el análisis factorial confirmatorio constató dicha estructura factorial. Por otro lado, el ISA demostró validez convergente al establecer correlaciones negativas con la Escala sobre Ideología de Género, tanto en hombres como en mujeres.

Respecto a la confiabilidad, el ISA evidenció índices de consistencia interna adecuados de .81 para el sexismo ambivalente, .84 (sexismo hostil) y .77 (sexismo benevolente) (De Lemus et al., 2008). A nivel de otros estudios, Garaigordobil et al. (2013a) también hallaron para el ISA valores altos de confiabilidad: .86 (SA), $.85(\mathrm{SH})$ y $.80(\mathrm{SB})$. Dicha tendencia se mantiene en adolescentes latinoamericanos al observarse índices Alfa de Cronbach de .89 (SA), 84 (SH) y .82 (SB) (Carbonell \& Mestre, 2019). En el presente estudio también se obtiene para el instrumento, índices adecuados de confiabilidad: Sexismo ambivalente (.87), sexismo hostil (.83) y sexismo benevolente (.79).

\section{Procedimiento}

El proyecto de investigación fue presentado a la coordinación de la Cátedra Unesco de Cultura y Educación para la Paz de la Universidad Técnica de Loja (UTPL), responsable de la intervención que realiza la institución en la comunidad de estudio. Tras obtener su aprobación, se procedió a coordinar la aplicación de los instrumentos durante los talleres de capacitación organizados por la Cátedra, empleándose diferentes estrategias de convocatoria para que la mayoría de adolescentes pudiera participar.

Durante el periodo de inscripción a los talleres, se solicitó el consentimiento informado de las madres/ padres para que sus hijos e hijas adolescentes pudieran participar del estudio. En el caso de los adolescentes, se les explicó y solicitó el asentimiento informado el mismo día la aplicación, antes de iniciar el proceso, brindándoles información sobre el objetivo de la investigación, el carácter voluntario de la participación, así como de la confidencialidad y anonimato de sus respuestas. A los participantes que aceptaron participar voluntariamente se les administró los instrumentos de evaluación. De esta manera, el estudio cumplió con los valores éticos requeridos en la investigación con seres humanos, respetando los principios fundamentales incluidos en la Declaración Helsinki.

\section{Análisis de datos}

Los datos recogidos fueron analizados mediante el paquete estadístico IBM SPSS (versión 22). En primer lugar, se evaluó el supuesto de normalidad de los datos utilizando la prueba Kolmogorov-Smirnov, y a partir de los resultados se procedió a utilizar pruebas paramétricas. Se realizaron los análisis estadísticos correspondientes de acuerdo a los objetivos de investigación utilizando el estadístico $\mathrm{t}$ de student para los contrastes de medias según el género. Las correlaciones entre el sexismo y las actitudes de justificación de la violencia se obtuvieron con el coeficiente de correlación de Pearson, siendo que las variables que establecieron una asociación moderada fueron consideradas para el análisis de regresión lineal. 


\section{RESULTADOS}

Sexismo y actitudes de justificación de la violencia

Tal y como se puede observar en la tabla 1, se confirman las asociaciones positivas y significativas entre el sexismo ambivalente, sus dimensiones y las actitudes de justificación del uso de la violencia entre iguales y violencia doméstica para la muestra total de adolescentes. Cabe destacar que las correlaciones más altas se establecen entre el sexismo ambivalente y hostil con ambos tipos de justificación.

\section{Tabla 1}

Correlaciones entre sexismo y actitudes de justificación de la violencia

\begin{tabular}{lccc}
\hline & Sexismo Ambivalente & Sexismo Hostil & Sexismo Benevolente \\
\hline Justificación de la violencia entre iguales & $.52^{* *}$ & $.59^{* *}$ & $.31^{* *}$ \\
Justificación de la violencia doméstica & $.58^{* *}$ & $.65^{* *}$ & $.36^{* *}$ \\
\hline
\end{tabular}

Nota: $* * \mathrm{p}<.01$

Al dividir la muestra según el género, se pudo observar que la tendencia hallada para la muestra general se mantiene solo en el grupo de las adolescentes mujeres. En el caso de los varones, las asociaciones positivas significativas solo se establecen entre el sexismo ambivalente y hostil con las actitudes de justificación de la violencia (ver tablas 2 y 3 ).

\section{Tabla 2}

Correlaciones entre sexismo y justificación de la violencia en adolescentes mujeres

\begin{tabular}{lccc}
\hline & Sexismo Ambivalente & Sexismo Hostil & Sexismo Benevolente \\
\hline Justificación de la violencia entre iguales & $.43^{* *}$ & $.48^{* *}$ & $.26^{*}$ \\
Justificación de la violencia doméstica & $.49 * *$ & $.58^{* *}$ & $.26^{*}$ \\
\hline
\end{tabular}

Nota: $* \mathrm{p}<.05, * * \mathrm{p}<.01$

\section{Tabla 3}

Correlaciones entre sexismo y justificación de la violencia en adolescentes varones

\begin{tabular}{lccc}
\hline & Sexismo Ambivalente & Sexismo Hostil & Sexismo Benevolente \\
\hline Justificación de la violencia entre iguales & $.44^{* *}$ & $.55^{* *}$ & .22 \\
Justificación de la violencia doméstica & $.50^{* *}$ & $.58^{* *}$ & .29 \\
\hline
\end{tabular}

Nota: $* * \mathrm{p}<.01$ 
De acuerdo a la evidencia presentada, la hipótesis de estudio 1 (un mayor nivel de sexismo estará asociado a un mayor nivel de justificación de la violencia entre iguales y violencia doméstica) se logra confirmar para la muestra total y para el grupo de las mujeres.

Variables predictoras de la justificación de la violencia entre iguales (JVI) y la violencia doméstica (JVD)

Tabla 4

Regresión lineal múltiple para la justificación de la violencia entre iguales

\begin{tabular}{lccccccc}
\hline & B & ETB & $\beta$ & $\mathrm{t}(67)$ & $\mathrm{R} 2$ & $\mathrm{~F}$ & $\mathrm{p}$ \\
\hline \multirow{3}{*}{ Sexismo hostil } & 7.92 & 1.26 & $.63^{* * *}$ & 6.30 & & & \\
Edad & 3.31 & 1.15 & $.29^{* *}$ & 2.88 & & & \\
\hline
\end{tabular}

Nota: $* * \mathrm{p}<.01, * * * \mathrm{p}<.001$

En relación a la JVD, se observa que el sexismo hostil por sí solo logra predecir positivamente sus puntajes, explicando el $40 \%$ de su variabilidad $(\mathrm{R} 2=.40, \mathrm{~F}=43.26, \mathrm{t}(67)=6.58, \mathrm{p}<.001, \mathrm{~B}=9.04, \beta=$ $.63, \mathrm{ETB}=1.38$ ).

Tras la evidencia presentada se puede concluir que se logra confirmar la hipótesis de investigación 2 (el sexismo hostil se comportará como un predictor positivo de las actitudes de justificación de la violencia).

\section{Diferencias por género}

Para el sexismo hostil (SH) se observó que el supuesto de igualdad de varianzas se cumplió $(\mathrm{F}=$ $1.53, \mathrm{p}>.05)$. De acuerdo a ello, se obtuvo que los adolescentes varones muestran significativamente $(\mathrm{t}(71)=-4.80, \mathrm{p}<.001, \mathrm{~d}=1.15)$ un mayor nivel de $\mathrm{SH}(\mathrm{M}=3.65, \mathrm{DE}=1.01)$, en contraste al grupo de mujeres $(\mathrm{M}=2.61, \mathrm{DE}=.85)$. En el caso del sexismo benevolente (SB), también se obtuvo igualdad de varianzas $(\mathrm{F}=2.40, \mathrm{p}>.05)$, observándose que los varones obtuvieron una mayor puntuación significativa en esta variable $(\mathrm{t}(71)=-2.58, \mathrm{p}<.05, \mathrm{~d}=.62, \mathrm{M}=$
Para este análisis, se introdujeron como variables dependientes ambos tipos de actitudes de justificación de la violencia y como variables predictoras al sexismo ambivalente y sus dimensiones, el género y la edad. En lo que concierne a la JVI, el sexismo hostil y la edad resultaron ser variables predictoras positivas de sus puntuaciones, explicando el 39\% de su variabilidad (ver tabla 4).
4.24, DE $=1.00$ ), en comparación a las adolescentes $(\mathrm{M}=3.70, \mathrm{DE}=0.79)$. De acuerdo a estos hallazgos, la hipótesis 3 (los varones presentarán mayor sexismo hostil y las mujeres mayores puntuaciones en sexismo benevolente) solo se cumple parcialmente, ya que el grupo de las adolescentes no obtuvo un mayor puntaje en SB.

$\mathrm{Al}$ evaluar las diferencias entre varones y mujeres en la justificación de la violencia, se obtuvo en primer lugar que, el supuesto de igualdad de varianzas no se cumplió, tanto en las puntuaciones de la violencia entre iguales $(\mathrm{F}=6.48, \mathrm{p}<.05)$ como en la violencia doméstica $(\mathrm{F}=6.25, \mathrm{p}<.05)$, por lo que el análisis de contraste tuvo que asumir heterogeneidad de varianzas. Los resultados para la JVI dieron cuenta de diferencias significativas, observándose puntuaciones superiores en los varones $(\mathrm{t}(52.03)=-2.93, \mathrm{p}<.01, \mathrm{~d}=.74, \mathrm{M}=$ $36.57, \mathrm{DE}=14.79)$ frente a las mujeres $(\mathrm{M}=27.16$, $\mathrm{DE}=11.45)$. Similar tendencia se observó para la JVD, donde los adolescentes obtuvieron una mayor puntuación en este tipo de actitud $(\mathrm{t}(47.03)=-3.15$, $\mathrm{p}<$ $.01, \mathrm{~d}=.81, \mathrm{M}=45.70, \mathrm{DE}=17.90)$, en contraposición a sus pares mujeres $(\mathrm{M}=33.88, \mathrm{DE}=12.06)$. 
En función a estos resultados se puede afirmar que se logra confirmar la última hipótesis del estudio (los adolescentes varones presentarán, respecto de las mujeres, mayores niveles de justificación de la violencia).

\section{DISCUSIÓN}

En las comunidades en riesgo psicosocial de América Latina, la interacción de una serie de factores (precaria situación socioeconómica y laboral, bajo acceso a servicios de educación, salud, vivienda, etc.) favorece el ejercicio de la violencia y dificulta los mecanismos de participación ciudadana que podrían potenciar el desarrollo sostenible de dichas comunidades. Por lo que se hace necesario identificar y promover el fortalecimiento de recursos en la comunidad que apuntalen los procesos de participación intersectorial y transformación social como estrategias en la prevención de la violencia. En ese sentido, el objetivo principal del estudio fue analizar las relaciones entre el sexismo (ambivalente, hostil y benevolente) y las actitudes de justificación del uso de la violencia (entre iguales y doméstica) en las y los adolescentes de una comunidad en riesgo psicosocial.

En primer lugar, los resultados corroboran la hipótesis de estudio 1, para la muestra total y en el grupo de mujeres, al evidenciarse relaciones positivas del sexismo ambivalente (SA), hostil ( $\mathrm{SH}$ ) y benevolente (SB) con la justificación de la violencia entre iguales (JVI) y violencia doméstica (JVD). Para el caso de los varones, las correlaciones solo se establecieron entre el SA, SH y ambos tipos de justificación de la violencia. Estos hallazgos en la muestra total son consistentes con estudios previos en otros contextos (Fasanelli et al., 2020; Ferragut et al., 2017; Garaigordobil et al., 2013a; Yamawaki et al., 2009) demostrando que en adolescentes de un contexto vulnerable, el sexismo ambivalente está asociado fuertemente a una mayor justificación de la violencia. No obstante, lo que resulta aún más preocupante es que para el caso de las mujeres se mantenga esta misma tendencia, puesto que esto podría suponer que algunas no llegan a reconocer el peligro en ciertas manifestaciones de violencia, por lo que las aceptan e incluso refuerzan. Así, la adherencia a las creencias sexistas y la tendencia hacia la justificación de la violencia, podrían estar relacionadas con la tendencia a minimizar la responsabilidad del agresor, en el caso de las mujeres víctimas de violencia (Fasanelli et al., 2020).

En esa línea, los resultados de los análisis de regresión que tenían como finalidad identificar las variables predictoras de las actitudes de justificación de la violencia en los y las adolescentes, confirman la hipótesis de estudio 2, revelando al sexismo hostil y a la edad como predictores positivos de la JVI. Asimismo, un mayor nivel de este tipo de sexismo se comportó como predictor de una mayor JVD. Por tanto, el sexismo hostil se comportaría como un predictor fuerte de las actitudes de justificación de la violencia, que presentan los y las adolescentes de esta comunidad en riesgo psicosocial. Dichos resultados también van en la línea de lo encontrado por Garaigordobil et al. (2013a) en adolescentes.

La violencia contra determinados grupos minoritarios se encuentra directamente relacionada con estereotipos (creencias negativas) y prejuicios en torno a los cuales se ha construido la identidad y que lamentablemente promueven la desigualdad y justificación de la violencia. Bajo la lógica de la cultura del honor, los varones establecen su honor basados en la virilidad, la protección de la familia y en la reputación como "hombre duro", mientras que el honor de las mujeres se centra en la vergüenza sexual (virginidad y restricciones sexuales). Desde esta perspectiva, la imagen pública de la familia, incluida la de la mujer, se convierte en un valor muy apreciado. Por tanto, las culturas que funcionan bajos los parámetros de la cultura del honor, suelen prestar una especial atención al honor del varón y a la regulación del comportamiento de la mujer, sobre todo el que está asociado a su sexualidad (Canto, San Martín, \& Perles, 2014).

Por tanto, la cultura del honor favorece el patrón patriarcal, dominante y machista, observándose que aún dichas características están muy presentes en comunidades Latinoamericanas como las del Ecuador y Perú. Para López-Zafra (2007) las sociedades que centran su desarrollo en la agricultura, suelen presentar valores materialistas que promueven la defensa de la propiedad, legitimando el uso de la violencia para defenderla. Este hecho podría dar cuenta de la mayor 
incidencia de la cultura del honor en países poco industrializados o en vías de desarrollo, donde el concepto de propiedad se extiende, no sólo a la tierra, sino también hacia las personas, particularmente las mujeres, por lo que este concepto nos puede ayudar a comprender un poco más el fenómeno de la violencia de género, que es un factor causal de la violencia de pareja.

Bajo este contexto, se promueven postulados que refuerzan y defienden los roles de género tradicionales que establecen una relación jerárquica entre varones y mujeres, siendo que los varones y "lo masculino" ocupan la posición dominante. Por lo que este tipo de organización social androcéntrica da sustento, en gran parte, al sexismo ambivalente (Canto et al., 2014). En ese sentido, la comunidad en riesgo psicosocial de donde proceden las y los adolescentes participantes podría presentar elementos de una estructura social que refuerza el sexismo, sobre todo el de tipo hostil, que predice en buena parte sus actitudes de justificación de la violencia doméstica y violencia entre iguales. En el caso de esta última, tener una mayor edad parece favorecer el fenómeno, debido probablemente a que las relaciones y conflictos con los pares o iguales emergen de manera más frecuente y adquieren una mayor importancia y protagonismo en esta etapa (Papalia et al., 2012).

En relación a las diferencias por género, en el caso del sexismo, los resultados indican que los adolescentes varones presentan un mayor nivel de $\mathrm{SH}$ y $\mathrm{SB}$, en contraste al grupo de mujeres. Esta evidencia nos permite concluir que la hipótesis 3 solo se cumple parcialmente, puesto que si bien se demuestra un mayor nivel de $\mathrm{SH}$ en los varones (Austin et al., 2019; Jitaru et al., 2017; Rottenbacher, 2010), las mujeres no llegan a destacar en el SB.

$\mathrm{Al}$ respecto, se podría sugerir que los adolescentes de esta comunidad en riesgo psicosocial, en comparación a sus pares mujeres, tendrían una mayor afinidad por la ideología de género tradicional y la defensa de los valores de una cultura del honor, razón por la cual podrían tener actitudes más hostiles con las mujeres que no asumen los roles de género tradicionales, y ser a su vez benevolentes con aquellas que consideran se ajustan más a un rol sumiso o poseen características positivas pero complementarias a ellos (Glick \& Fiske, 2001; Rodríguez et al., 2009).

En cuanto a la justificación del uso de la violencia, los varones obtuvieron una mayor puntuación en contraste a sus pares mujeres, lo cual podría estar relacionado al resultado anterior, si se considera que el sexismo se comporta como un predictor de las actitudes de justificación de la violencia. En ese sentido, este resultado podría estar dando cuenta del alto nivel de creencias relacionadas a la dominación patriarcal y sexista que presentan los adolescentes varones (DíazAguado et al., 2004).

Dentro de esta línea de interpretación, también es importante considerar el papel de los prejuicios asociados a la estigmatización individual y social de la comunidad. Los cuales podrían movilizar en los adolescentes una tendencia a compensar la situación de estigmatización percibida como injusta, a través del desarrollo de actitudes que justifican el uso de la violencia al resolver los conflictos en sus relaciones (Jhangiani et al., 2014). Es preciso recordar que la comunidad de donde provienen los participantes presenta una serie de problemáticas que al no ser gestionadas eficazmente han contribuido a generar una percepción estigmatizada del barrio. Finalmente, cabe resaltar que este hallazgo resulta similar a lo encontrado en estudios previos (Garaigordobil et al., 2013b; Pinos et al., 2016) lo que confirma la hipótesis de estudio 4.

A la luz de estos hallazgos, se puede concluir que el sexismo hostil, en contraste al sexismo benevolente, presenta un mayor poder al predecir las actitudes que justifican el uso de la violencia entre iguales y violencia doméstica en los y las adolescentes de esta comunidad en riesgo psicosocial. Asimismo, las relaciones halladas para el grupo de las mujeres, respecto al sexismo ambivalente y la justificación de la violencia, así como el reporte de mayores puntuaciones de esta variable y del sexismo en los adolescentes varones, se pueden identificar como elementos de riesgo para el ejercicio de la violencia de pareja, por lo que es importante desarrollar un programa de prevención diferenciado por género.

Esta investigación presenta algunas limitaciones las cuales son importantes mencionar a fin de que los 
resultados sean interpretados considerando su posible influencia. En primer lugar, los resultados hallados sobre las asociaciones entre las variables no pueden ser generalizados a la población de adolescentes en riesgo psicosocial de Ecuador. Por otro lado, el uso de auto informes puede requerir una cierta capacidad de introspección e implicar un riesgo de deseabilidad social. Por último, el diseño de investigación empleado no permite realizar afirmaciones con mayor profundidad en cuanto a la relación de variables, por lo que se recomienda para posteriores estudios utilizar ecuaciones estructurales con un mayor número de participantes y considerando otras variables comunitarias y de contex to asociadas a la problemática.

\section{IMPLICACIONES PRÁCTICAS}

Sobre la base de lo hallado y teniendo en cuenta la prevalencia de los casos de violencia en esta comunidad en contra de las mujeres, niños y niñas, $y$ en el contexto de pareja, es que se sugiere plantear un conjunto de acciones que deriven en un programa comunitario de prevención de la violencia de pareja, el cual se integre al modelo de desarrollo sostenible de la comunidad. Este programa implicaría la gestión de acciones desde y para los y las adolescentes, que contribuya a la prevención de la violencia a nivel relacional, ya que según algunas investigaciones existe una relación positiva moderada entre la violencia entre iguales y la violencia de pareja en adolescentes (Cava, Buelga, \& Tomás, 2018; Vivolo-Kantor, O’Malley, \& Bacon, 2016).

Los fundamentos teóricos que sustenten el programa podrían contemplar la perspectiva de género, ya que muchos casos de violencia encuentran su origen en la asimetría relacional y desigualdad de género, y se producen con mayor frecuencia en contra de las mujeres (Echeburúa, 2019; Ferrer \& Bosch, 2019). Pero también, la Teoría de Conflictos de Galtung, puesto que aporta a la comprensión del fenómeno y brinda un posible método de intervención desde la promoción de la cultura de paz (Arriaga, 2013).

En esa línea, la propuesta del programa podría incluir la construcción conjunta (adolescentes, comunidad, instituciones, profesionales) de espacios de diálogo fundamentados en la teoría de resolución de conflictos y con enfoque de género, en los cuales se promueva la reflexión y discusión del impacto que puede tener la cultura del honor, el sexismo y las actitudes que justifican la violencia versus la cultura de paz, el respeto por los derechos humanos y la igualdad de género, en las relaciones sociales, proyecto de vida personal y el desarrollo comunitario.

En este proceso, el desarrollo del pensamiento crítico, la capacidad de razonamiento y la asertividad serían habilidades clave que podrían viabilizar dicho intercambio. Asimismo, a través de la creatividad se podría hallar posibles soluciones no-violentas al conflicto relacional y la desigualdad de género, promoviendo un escenario en donde cada una de las partes obtuviera resultados favorables y paz positiva, la cual implica justicia, y por ende, la ausencia de violencia (Arriaga, 2013). Según algunos estudios, las dificultades para la resolución positiva de conflictos, o la carencia de habilidades de comunicación podrían favorecer la implicación de los y las adolescentes en el ejercicio de la violencia entre iguales y violencia de pareja (Carrascosa et al., 2019; González-Ortega et al., 2008).

Por tanto, el programa podría plantearse como objetivos: promover a través del diálogo y la participación reflexiva (bajo un enfoque de género y la teoría de resolución de conflictos) la potenciación de la creatividad, pensamiento crítico, capacidad de razonamiento y asertividad en las y los adolescentes, para que logren una mayor identificación con los valores de la cultura de paz y con la igualdad de género, y asuman acciones destinadas a reducir el sexismo y las actitudes que justifican el uso de la violencia a nivel individual y en su comunidad.

Es muy importante que el programa base sus acciones en los principios de libertad, pluralidad y flexibilidad, por lo que la actuación de las y los profesionales tendría que enfocarse en dinamizar la intervención a través de la generación de procesos participativos y de trasmisión de conocimientos técnicos (principios del enfoque de género, de la teoría de conflictos, de la intervención social comunitaria, habilidades sociales, emprendimiento, etc.) que la comunidad necesita para potenciar su desarrollo (Marchioni, 2001). 
Esta intervención de prevención de la violencia al ser parte del modelo de desarrollo sostenible de la comunidad, tendría que plantearse lograr una articulación de acciones con las asociaciones de participación ciudadana, los centros educativos, y los servicios de salud y de seguridad de la zona, guiados bajo la metodología de acción social y comunitaria. $\mathrm{Y}$ durante el proceso, redefinir sus estrategias para implicar directa y progresivamente a toda la comunidad, considerando sus recursos y factores de riesgo.

El apoyo proveniente de la Cooperación al Desarrollo puede ser un actor clave en este proceso, puesto que su objetivo es promover de forma sostenible y equitativa el progreso económico, social y global de los países y comunidades en vías de desarrollo. Específicamente la Cooperación al Desarrollo proveniente de la Academia, socio estratégico en este proceso, aporta al modelo de desarrollo sostenible de esta comunidad a través de la cooperación técnica, la cual implica un intercambio de conocimientos técnicos y de gestión que promueven la potenciación de las capacidades de la población y sus instituciones. No obstante, es vital que las y los profesionales vinculados a dicha cooperación puedan transmitir y gestionar adecuadamente su verdadero propósito, a fin de evitar que la comunidad y sus líderes polaricen sus posiciones, caigan en servilismo o se dificulte su proceso de empoderamiento.

\section{REFERENCIAS}

Aguirre, M., Moreira, D., Zavaleta, L., \& Pérez, S. J. (2019). Intervención psicosocial con niños en contextos de vulnerabilidad. El caso del barrio Tierras Coloradas, Loja - Ecuador. Educació Social. Revista d'Intervenció Socioeducativa, 72, 77-101.

Albarracín, D., Johnson, B. T., \& Zanna, M. P. (Eds.). (2005). The handbook of attitudes (pp. 223-271). Mahwah, NJ: Lawrence Erlbaum.

Archer, J. (2006). Cross-cultural differences in physical aggression between part-ners: A social role analysis. Personality and Social Psychology Review, 10, 113 -133. http://doi.org/10.1207/ s15327957pspr1002 3
Arnoso, A., Ibabe, I., Arnoso, M., \& Elgorriaga, E. (2017). El sexismo como predictor de la violencia de pareja en un contexto multicultural. Anuario de Psicología Jurídica, 27, 9-20

Arriaga, N. (2013). La ética en la transformación de conflictos a partir de la propuesta de Johan Galtung: el caso de violencia de género (Tesis de Maestría, Universidad Autónoma del Estado de México, UAEM) http://ri.uaemex.mx/ handle/20.500.11799/49440

Austin, D., \& Jackson, M. (2019). Benevolent and hostile sexism differentially predicted by facets of right-wing authoritarianism and social dominance orientation. Personality and Individual Differences, 139, 34-38.

Calderón, P. (2009). Teoría de conflictos de Johan Galtung. Revista Paz y Conflictos, 2, 60-81.

Canto, J. M., San Martín, J., \& Perles, F. (2014). La relación entre la cultura del honor y otras variables de carácter ideológico. Boletín de Psicología, 111, 57-69.

Carbonell, A., \& Mestre, M.V. (2019). Sexismo, amor romántico y desigualdad de género. Un estudio en adolescentes latinoamericanos residentes en España. América Latina Hoy, 83, 59-74. https:// doi.org/10.14201/alh2019835974

Carrascosa, L., Cava, M. J., Buelga, S., \& de Jesus, S. (2019). Reduction of sexist attitudes, romantic myths, and aggressive behaviors in adolescents: efficacy of the DARSI program. Psicothema, 31(2), 121-127.

Cava, M. J., Buelga, S., \& Tomás, I. (2018). Peer victimization and dating violence victimization: the mediating role of loneliness, depressed mood, and life satisfaction. Journal of Interpersonal Violence, 1-26. https://doi. org/10.1177/0886260518760013.

De Lemus, S., Castillo, M., Moya, M., Padilla, J. L., \& Ryan, E. (2008). Elaboración y validación del Inventario de Sexismo Ambivalente para Adolescentes. International Journal of Clinical and Health Psychology, 8(2), 537-562.

Díaz-Aguado, M. J., Martínez, R., \& Martín, G. (2004). Prevención de la violencia y lucha contra la exclusión desde la adolescencia. La violencia entre iguales en la escuela y en el ocio. Estudios 
comparativos e instrumentos de evaluación. Instituto de la Juventud.

Durán, M., Moya, M., Megías, J. L., \& Viki, T. (2010). Social perception of rape victims in dating and married relationships: The role of perpetrator's benevolent sexism. Sex Roles, 62, 505-519.

Echeburúa, E. (2019). Sobre el papel del género en la violencia de pareja contra la mujer. Comentario a Ferrer-Pérez y Bosch-Fiol, 2019. Anuario de Psicología Jurídica, 29, 77-79.

Fasanelli, R., Galli, I., Grassia, M. G., Marino, M., Cataldo, R., Lauro, C., Castiello, C., Grassia, F., Arcidiacono, C., \& Procentese, F. (2020). The Use of Partial Least Squares-Path Modelling to Understand the Impact of Ambivalent Sexism on Violence-Justification among Adolescents. International journal of environmental research and public health, 17(14), 4991. https://doi. org/10.3390/ijerph17144991

Ferragut, M., Blanca, M. J., Ortiz-Tallo, M., \& Bendayan, R. (2017). Sexist attitudes and beliefs during adolescence: A longitudinal study of gender differences. European Journal of Developmental Psychology, 14(1), 32-43. https://doi.org/10.1080 /17405629.2016.1144508

Ferrer-Pérez, V., \& Bosch-Fiol, E. (2019). El género en el análisis de la violencia contra las mujeres en la pareja: de la "Ceguera" de género a la investigación específica del mismo. Anuario de Psicología Jurídica, 29, 69-76. https://doi. org/10.5093/apj2019a3

Garaigordobil, Maite. (2014). Sexism and empathy: Differences as a function of sociodemographic variables and relations between both constructs. Advances in Psychology Research, 100, 59 - 80.

Garaigordobil, M., \& Aliri, J. (2013a). Relaciones del sexismo con justificación de la violencia, y con otras formas de prejuicio como la dominancia social y el autoritarismo. Estudios de Psicología, 34 (2), 127-139.

Garaigordobil, M., Aliri, J., \& Martínez-Valderrey, V. (2013b). Justificación de la violencia durante la adolescencia: Diferencias en función de variables sociodemográficas. European Journal of Education and Psychology, 6(2), 83-93.
Glick, P., \& Fiske, S. T. (1996). The ambivalent sexism inventory: Differentiating hostile and benevolent sexism. Journal of Personality and Social Psychology, 70, 491-512. https://doi. org/10.1037/0022-3514.70.3.491.

Glick, P., \& Fiske, S. T. (2001). An ambivalent alliance: Hostile and benevolent sexism as complementary justifications for gender inequality. American Psychologist, 56, 109-118. https://doi. org/10.1037/0003-066x.56.2.109.

Glick, P., Fiske, S. T., Mladinic, A., Saiz, J. L., Abrams, D., Masser, B., ..., \& López, W. (2000). Beyond prejudice as simple antipathy: Hostile and benevolent sexism across cultures. Journal of Personality and Social Psychology,79(5), 763775. https://doi.org/10.1037/0022-3514.79.5.763

Glick, P., Sakalli-Ugurlu, N., Ferreira, M. C., \& Aguiar de Souza, M. (2002). Ambivalent sexism and attitudes toward wife abuse in Turkey and Brazil. Psychology of Women Quarterly, 26, 292-297.

González-Ortega, I., Echeburúa, E., \& de Corral, P. (2008). Variables significativas en las relaciones violentas en parejas jóvenes: una revisión. Behavioral Psychology, 16(2), 207-225.

Heise L., \& García-Moreno, C. (2002). Violence by intimate partners. En E.G. Krug, L.L. Dahlberg, J.A. Mercy, A.B. Zwi y R. Lozano (dirs.), World report on violence and health (pp. 87-121). Ginebra: World Health Organization.

Herrero, J., Rodríguez, F., \& Torres, A. (2017). Acceptability of Partner Violence in 51 Societies: The Role of Sexism and Attitudes Toward Violence in Social Relationships. Violence Against Women, 23(3) 351-367.

Ibabe,I.,Arnoso,A., \& Elgorriaga,E.(2016).Ambivalent sexism inventory: Adaptation to Basque population and sexism as a risk factor of dating violence. Spanish Journal of Psychology, 19, 1-9.

Instituto Nacional de Estadísticas y Censos de Ecuador. (2018). Atlas de Género. https://www. ecuadorencifras.gob.ec/documentos/web-inec/ Bibliotecas/Libros/Atlas_de_Genero_Final.pdf

Jackson, L. M. (2011). The psychology of prejudice: From attitudes to social action. American Psychological Association 
Jhangiani, R., \& Tarry, H. (2014) Principles of Social Psychology - 1st International Edition. Victoria, B.C.: BCcampus. https://opentextbc.ca/ socialpsychology/

Jitaru, M., \& Holman, A. C. (2017). Ambivalent sexism in Romanian students: Examining gender, sex roles and social dominance. Annals of A. I. I. Cuza University. Psychology Series, 26(1), 45-59.

Lee, T., Fiske, S. T., Glick, P., \& Chen, Z. (2010). Ambivalent Sexism in Close Relationships: (Hostile) Power and (Benevolent) Romance Shape Relationship Ideals. Sex Roles, 62(7-8), 583-601. https://doi.org/10.1007/s11199-010-9770-x

León-Ramírez, B., \& Ferrando, P. J. (2014). Assessing sexism and gender violence in a sample of Catalan university students: A validity study based on the Ambivalent Sexism Inventory and the Dating Violence Questionnaire. Anuario de Psicologíal The UB Journal of Psychology, 44, 327-341.

López-Zafra, E. (2007). Elaboración de una escala para medir Cultura del Honor. Revista de Psicología Social, 22(1), 31-42. https://doi. org/10.1174/021347407779697520

Malonda, E. (2014). El sexismo en la adolescencia. Factores Psicosociales moduladores (Tesis Doctoral, Universidad de Valencia). http://roderic. uv.es/handle/10550/36213

Marchioni, M. (2001). Comunidad y cambio social. Teoría y praxis de la acción comunitaria. Popular.

OMS. (29 de noviembre de 2017). Violencia contra la mujer. Datos y cifras. https://www.who.int/es/ news-room/fact-sheets/detail/violence-againstwomen

Papalia, D. E., Feldman, R. D., \& Martorell, G. (2012). Desarrollo Humano. México D.C: McGraw-Hill. https://www.academia.edu/20423118/Desarrollohumano-Papalia-12a

Pinos, V., Pinos, G., Jerves, M., \& Enzlin, P. (2016). Attitudes of adolescents toward violence and their related factors. MASKANA, 7(2), 1-15.

Ramiro-Sánchez, T., Ramiro, M. T., Bermúdez, M. P.,
\& Buela-Casal, G. (2018). Sexism in adolescent relationships: A systematic review. Psychosocial Intervention, 27, 123-132.https://doi.org/10.5093/ pi2018a19

Rodríguez, Y., Lameiras, M., Carrera, M. V., \& Faílde, J. M. (2009). Aproximación conceptual al sexismo ambivalente: Estado de la cuestión. SUMMA Psicológica UST, 6(2), 131-142.

Rose, A. J., \& Rudolph, K. D. (2006). A review of sex differences in peer relationship processes: Potential trade-offs for the emotional and behavioral development of girls and boys. Psychological Bulletin, 132, 98-131. https://doi. org/10.1037/0033-2909.132.1.98

Rottenbacher, J. M. (2010). Sexismo ambivalente, paternalismo masculino e ideología política en adultos jóvenes de la ciudad de lima. Pensamiento Psicológico, 7(14), 9-18.

Sardinha, L., \& Nájera, H. E. (2018). Attitudes towards domestic violence in 49 low- and middle-income countries: A gendered analysis of prevalence and country-level correlates. PLoS ONE, 13(10), 1-18.

UNICEF. (2014). La niñez y adolescencia en el Ecuador contemporáneo: avances y brechas en el ejercicio de derechos. https://www.unicef.org/ ecuador/NA_Ecuador_Contemporaneo.pdf

Vivolo-Kantor, A. M., O’Malley, E., \& Bacon, S. (2016). Associations of teen dating violence victimization with school violence and bullying among US high school students. Journal of School Health, 86(8), 620-627.

Yamawaki, N., Ostenson, J., \& Brown, C. R. (2009). The functions of gender role traditionality, ambivalent sexism, injury, and frequency of assault on domestic violence perception. Violence Against Women, 15, 1126-1142.

Zawadzki, M., Shields, S. A., Danube, C. L., \& Swim, J. (2013). Using WAGES to raise awareness of and reduce endorsement of sexism via experiential learning. Psychology of Women Quarterly, 67(1112), 605-616.

Fecha de recepción: 25 de agosto 2020

Fecha de aceptación: 5 de octubre 2020 